\title{
Percepción materna del estado nutricional de sus hijos que acuden a un consultorio pediátrico, Asunción, 2018
}

\author{
Maternal perception of the nutritional status of their \\ children who come to a pediatric office, Asunción, 2018
}

\author{
Leticia Ramirez Pastore`, Sylvia Gotz ${ }^{1}$, Victor Guillermo Sequera², Jacinto Riera', Brunilde \\ Pastore ${ }^{1}$, Norma Vera', Liz Castaño
}

\section{RESUMEN}

Introducción: La percepción de la madre acerca del estado nutricional de su hijo es clave para el éxito o fracaso de las medidas preventivas o terapéuticas a implementar en relación a la obesidad. El objetivo de este trabajo fue evaluar la concordancia entre la percepción de las madres acerca del estado nutricional de sus hijos y su estado nutricional real. Materiales y Métodos: Estudio observacional, descriptivo con componente analítico, trasversal. La percepción materna del estado nutricional del hijo fue comparada con el índice de masa corporal real. Los datos fueron expresados en porcentajes. La concordancia se determinó a través del coeficiente Kappa de Cohen $(p \leq 0,05)$. Para evaluar los factores asociados con la falla de la madre en percibir correctamente el estado nutricional de su hijo se realizó el análisis de regresión logística bivariada y mutivariado con los factores significativos. Resultados: Fueron evaluados 636 pacientes, 54\% masculinos, con edad media de 10 años. Se encontraban con peso adecuado $57 \%$, bajo peso $9 \%$, sobrepeso $22 \%$ y obesidad $11 \%$. El $59,9 \%$ de las madres clasificaron correctamente el estado nutricional de su hijo, kappa 0,09 ( $p=0,0003)$. Los factores asociados significativamente con la falla de la madre en percibir correctamente el estado nutricional de su hijo fueron: el sexo masculino (OR 1,54), edad entre 4 a 9 años (OR 1,92), edad entre 9 a 14 años (OR 1,23) e Indice de Masa Corporal - Z score para edad y sexo (OR 2,04). El análisis multivariado mostró valores similares $(p \leq 0,001)$. Conclusión: Aproximadamente el $60 \%$ de las madres clasifican el estado nutricional de sus hijos de manera correcta. Sin embargo, los niños con sobrepeso y obesidad

\section{ABSTRACT}

Introduction: The mother's perception regarding the nutritional status of her child is a key to the success or failure to implement preventive or therapeutic measures to manage obesity. The objective of this study was to evaluate the concordance between mothers' perception of the nutritional status of their children and their actual nutritional status. Materials and Methods: This was an observational, descriptive and cross-sectional study with an analytical component. The maternal perception of the child's nutritional status was compared with the actual body mass index. The data were expressed in percentages. Concordance was determined through Cohen's Kappa coefficient $(\mathrm{p} \leq 0.05)$. To assess the factors associated with the failure of the mother to correctly perceive the nutritional status of her child, the bivariate and multivariate logistic regression analysis was performed with the significant factors. Results: A total of 636 patients were evaluated, $54 \%$ were male, the average age was 10 years. $57 \%$ had an adequate weight, 9\% were underweight, $22 \%$ were overweight and $11 \%$ had obesity. $59.9 \%$ of the mothers correctly classified the nutritional status of their child, kappa $0.09(\mathrm{p}=0.0003)$. Factors that were significantly associated with the failure of the mother to correctly perceive the nutritional status of her son were: male sex (OR 1.54), age between 4 to 9 years old (OR 1.92), age between 9 to 14 years old (OR 1.23) and Body Mass Index - Z score for age and sex (OR 2.04). The multivariate analysis showed similar values $(\mathrm{p} \leq 0.001)$. Conclusion: Approximately $60 \%$ of mothers correctly classified the nutritional status of their children. However, overweight and obese children were recognized by their mothers as

\footnotetext{
${ }^{1}$ Centro Pediátrico Integral Riera SRL. Asunción. Paraguay.

${ }^{2}$ Ministerio de Salud Pública y Bienestar Social, Dirección Nacional de Vigilancia Sanitaria. Asunción, Paraguay.

Correspondencia: Leticia Ramírez Pastore; Correo: letty.ramirez@hotmail.com

Conflicto de interés: Los autores declaran no poseer conflicto de interés

Recibido: 13/11/2018; Aceptado: 12/12/2018

DOI: https://doi.org/10.31698/ped.45032018005
} 
son reconocidos por sus madres como tales sólo en un 31\% de los casos. Se considera estos hallazgos como de gran importancia para el éxito de los programas de intervención en obesidad.

Palabras clave: Estado nutricional, percepción de peso, obesidad infantil.

\section{INTRODUCCIÓN}

La prevalencia de sobrepeso y obesidad infantil está aumentando de manera muy rápida a nivel mundial ${ }^{(1)}$, así como en Latinoamérica y Paraguay. Constituye una llamada de atención clave para los pediatras ya que predispone a un número creciente de niños y adolescentes con mayor riesgo de problemas tanto médicos ${ }^{(2,3)}$ como sociales, tales como dislipidemias ${ }^{(4,5)}$, presión arterial elevada ${ }^{(5)}$, diabetes $^{(6)}$, esteatohepatitis ${ }^{(7)}$, apnea del sueño ${ }^{(8)}$, trastornos ortopédicos ${ }^{(9)}$, aislamiento social, depresión, entre otros.

Es importante reconocer a aquellos niños que se encuentran con sobrepeso u obesidad, a fin de implementar estrategias de prevención y tratamientos adecuados desde las edades tempranas de la vida.

Estudios preliminares ${ }^{(10,11)}$ sobre la percepción de la madre acerca del estado nutricional de su hijo revelan la importancia de la concienciación acerca del tema y su relación directa con el éxito o fracaso de las medidas preventivas o terapéuticas a implementar.

Específicamente la madre tiene un rol primordial en la formación de los estilos de vida, ya que es ella quien representa el principal agente de cuidado y crianza de los hijos, por tanto se considera como mediadora en los esfuerzos para prevenir el sobrepeso y obesidad infantil, actuando como modeladora de los patrones de alimentación y ejercicio ${ }^{(12,13)}$, rol que en ciertas culturas es aún más reforzado.

Sin embargo, estudios revelan que las madres no perciben cuando su hijo presenta sobrepeso $\mathrm{u}$ obesidad $^{(14)}$, y tienden a subestimarlo, incluso cuando el hijo se encuentra con peso adecuado ${ }^{(15)}$. such in only $31 \%$ of cases. These findings are considered to be of great importance for the success of obesity intervention programs.

Keywords: Nutritional status, weight perception, childhood obesity

Es difícil que cualquier estrategia funcione de la manera esperada si las madres de los niños no son capaces de reconocer en sus hijos el exceso de peso y de masa grasa, así como los factores de riesgo asociados y sus consecuencias.

El objetivo de este trabajo es evaluar la concordancia entre la percepción de las madres acerca del estado nutricional de sus hijos y el estado nutricional real de los mismos.

\section{MATERIAL Y MÉTODOS}

Estudio observacional, descriptivo con componente analítico, de corte trasversal, llevado a cabo en un consultorio pediátrico privado al inicio del año escolar del año 2018.

Fueron evaluados los pacientes de ambos sexos, de 4 a 19 años edad, que acudieron a consulta de niño sano y chequeo médico previo al inicio de clases, de enero a marzo de 2018.

La percepción materna del estado nutricional de los hijos se midió a través de una encuesta con la siguiente pregunta a la madre: "En cuanto al estado nutricional de su hijo, ¿en qué categoría le parece que se encuentra? Bajo Peso/Peso Adecuado/ Sobrepeso/Obesidad" ${ }^{(16)}$. Se midió el peso y la talla de cada paciente con una balanza y tallímetro de precisión (SECA) y se determinó el estado nutricional real de cada niño según las tablas de Índice de Masa Corporal Z-Score (BAZ) para edad y sexo de la OMS 2007 que define en estado nutricional adecuado a aquellos pacientes con BAZ entre $-1 \mathrm{a}+1$ $\mathrm{DE}$, bajo peso a aquellos con BAZ menor a $-1 \mathrm{DE}$, sobrepeso a aquellos con BAZ entre +1 y +2 DE y 
obesidad a aquellos con BAZ mayor a +2DE. Además, se le preguntó a la madre su peso y altura para posteriormente calcular también su índice de masa corporal (IMC) y estado nutricional, definiendo estado nutricional adecuando al IMC entre 18,5-24,9, bajo peso al IMC menor a 18,5, sobrepeso al IMC entre 25 y 29,9 y obesidad al IMC igual o mayor a 30 .

El tamaño de muestra fue calculado en base al porcentaje de obesidad en niños en Latinoamérica $(2016)^{(17)}$ con una proporción esperada de $17 \%$, error de 0,05 y un intervalo de confianza de $95 \%$, con un total de 217 pacientes.

De 891 pacientes evaluados, quedaron incluidos 636 pacientes y fueron descartados 255 debido a que no acudieron a la consulta con la madre o la ficha no se encontraba completa. Llama la atención 76 fichas donde el único dato faltante era el peso de la madre, no así su altura y la percepción de peso de su hijo.

Los datos fueron analizados utilizando Stata v12. Los datos fueron expresados en porcentajes. La concordancia se determinó a través del coeficiente Kappa de Cohen y se consideró significativa una $\mathrm{p} \leq 0,05$. Para evaluar los factores asociados con la falla de la madre en percibir correctamente el estado nutricional de su hijo se realizó el análisis de regresión logística bivariada y luego el análisis mutivariado con los factores que resultaron estadísticamente significativos.

\section{RESULTADOS}

Fueron evaluados 636 pacientes, $54,4 \%$ de sexo masculino, con una edad promedio de 10 años, valor mínimo de 4 años, máximo de 19 años.

De ellos, se encontraban con peso adecuado el $57,23 \%$ de los pacientes, con bajo peso el $9,28 \%$, sobrepeso $22,17 \%$ y obesidad $11,32 \%$ de ellos.

En cuanto al estado nutricional de las madres, se encontraban con peso adecuado $67,14 \%$ de ellas, con bajo peso $0,94 \%$, sobrepeso $22,8 \%$ y obesidad $9,12 \%$. (Tabla 1).

Al analizar el estado nutricional de los pacientes según sexo y grupos de edad (Tabla 2), se observa una disminución de los porcentajes de sobrepeso y obesidad a mayor edad, así mismo un mayor porcentaje de pacientes con peso adecuado en el rango entre 14 a 19 años de edad. En cuanto al bajo peso, a mayor edad, menor porcentaje de pacientes con bajo peso entre los varones, pero más mujeres con bajo peso en el rango de mayores de 14 años.

Tabla 1. Características descriptivas de la población.

\begin{tabular}{lc}
\hline Variables & $\mathbf{n}(\%)$ \\
\hline Sexo & $346(54.4)$ \\
$\quad$ Masculino & $290(45.6)$ \\
Femenino & \\
Edad (años) & $252(39.62)$ \\
4 a $<9$ & $250(39.31)$ \\
9 a $<14$ & $134(21.07)$ \\
14 a 19 & \\
Estado Nutricional de pacientes (BAZ) & $59(9.28)$ \\
Bajo Peso & $364(57.23)$ \\
Peso Adecuado & $141(22.17)$ \\
Sobrepeso & $72(11.32)$ \\
Obesidad & \\
Estado Nutricional de la madre (IMC) & $6(0.94)$ \\
Bajo Peso & $427(67.14)$ \\
Peso Adecuado & $145(22.8)$ \\
Sobrepeso & $58(9.12)$ \\
Obesidad & \\
\hline Baz: Indice de masa corporal Z-score para sexo y edad & \\
IMC: Indice de Masa Corporal
\end{tabular}

Tabla 2. Distribución del estado nutricional de los pacientes según el sexo y edad.

\begin{tabular}{|c|c|c|c|c|c|c|c|c|c|}
\hline \multicolumn{10}{|c|}{ Estado nutricional de los pacientes $\mathbf{n}(\%)^{*}$} \\
\hline & \multicolumn{2}{|c|}{ Bajo Peso } & \multicolumn{2}{|c|}{ Peso Adecuado } & \multicolumn{2}{|c|}{ Sobrepeso } & \multicolumn{2}{|c|}{ Obesidad } & \multirow[b]{2}{*}{ TOTAL } \\
\hline Edad (años) & Femenino & Masculino & Femenino $\mathrm{N}$ & Masculino & o Femenino & o Masculino & Femenin & o Masculino & \\
\hline $4 a<9$ & $5(2)$ & $20(7.9)$ & $63(25)$ & $69(27.4)$ & ) $28(11.1)$ & $32(12.7)$ & $9(3.6)$ & $26(10.3)$ & $252(100)$ \\
\hline $9 a<14$ & $6(2.4)$ & $17(6.8)$ & $72(28.8)$ & $69(27.6)$ & $29(27.6)$ & 29 (11.6) & $12(4.8)$ & $16(6.4)$ & $250(100$ \\
\hline$>14$ & $8(6)$ & $3(2.2)$ & $46(34.3)$ & $45(33.6)$ & $11(8.2)$ & $12(9)$ & $1(0.7)$ & $8(6)$ & $134(100)$ \\
\hline
\end{tabular}

$\mathrm{Al}$ analizar los datos del estado nutricional real de la madre y el estado nutricional real del hijo, se observa un $49,69 \%$ de concordancia, con un coeficiente kappa de 0,09 (concordancia pobre) y $\mathrm{p}=0,0003$. Los pacientes obesos tienen madres obesas o con sobrepeso en un $51,4 \%$ de los casos, aquellos con sobrepeso tienen madres con sobrepeso u obesidad en un $38,3 \%$ de los casos, mientras que aquellos con peso adecuado tienen madres con sobrepeso $\mathrm{u}$ obesidad en un $27,2 \%$ de los casos.

La comparación entre la percepción materna del estado nutricional de su hijo y el estado nutricional real se muestra en la Tabla 3. 
Tabla 3. Percepción materna del estado nutricional de sus hijos.

\begin{tabular}{lcccc}
\hline \multicolumn{5}{c}{ Estado nutricional de los pacientes n (\%)* } \\
\hline Percepción & Bajo Peso & Peso Adecuado & Sobrepeso & Obesidad \\
Bajo Peso & $19(32.2)$ & $34(9.3)$ & $2(1.4)$ & $2(0.08)$ \\
Peso Adecuado & $40(67.8)$ & $325(89.3)$ & $109(77.3)$ & $30(41.7)$ \\
Sobrepeso & 0 & $5(1.4)$ & $30(21.3)$ & $33(45.8)$ \\
Obesidad & 0 & 0 & 0 & $7(9.7)$ \\
TOTAL & $59(100)$ & $364(100)$ & $141(100)$ & $72(100)$ \\
\hline
\end{tabular}

*Estado nutricional definido según el Z-score de Indice de Masa Corporal para edad y sexo.

Casi $3 / 5(59,9 \%)$ de las madres clasificaron correctamente el estado nutricional de su hijo. El coeficiente de concordancia kappa fue de 0,22 (concordancia débil), con un error estándar de 0,02 y $\mathrm{p} \leq 0,0001$.

Los pacientes con bajo peso fueron clasificados correctamente por sus madres en un $32,2 \%$ de los casos; aquellos con peso adecuado fueron clasificados correctamente en un $89,3 \%$ de los casos; los que tenían sobrepeso en un $21,3 \%$ de los casos y los obesos fueron clasificados correctamente en un $9,7 \%$ de los casos.

Los pacientes clasificados incorrectamente constituyeron el 40,1\% de los casos. Los pacientes con bajo peso fueron clasificados incorrectamente siempre como con "peso adecuado". Los pacientes con peso adecuado fueron clasificados como con "bajo peso" en un $9,3 \%$ fueron clasificados como con "peso adecuado" en un $89,3 \%$ de los casos y nunca como obesos, mientras que los obesos fueron clasificados como con "peso adecuado" en un $41,7 \%$ de los casos o con "sobrepeso" en un $45,8 \%$ de los casos.

$\mathrm{Al}$ analizar exclusivamente los datos discordantes, se obtuvo los siguientes resultados. Los datos discordantes corresponden en un $61,2 \%$ a pacientes de sexo masculino ( $\mathrm{p}=0,005), 47 \%$ a pacientes de $4-9$ años, 36\% de 9-14 años y 17\% de 14-19 años $(p=0,005)$. En cuanto al estado nutricional de los pacientes, hay menor discordancia en el peso adecuado con un $15,3 \%$, mientras que aquellos con sobrepeso representan el $43,5 \%$ y los obesos el $25,5 \%$ de los discordantes $(\mathrm{p} \leq 0,0001)$.

Los odds ratio calculados del análisis de regresión logística bivariado y multivariado se muestran en la Tabla 4. Los factores asociados significativamente con la falla de la madre en percibir correctamente el estado nutricional de su hijo fueron el sexo masculino (OR 1,54), edad entre 4 a 9 años (OR 1,92), edad entre 9 a 14 años (OR 1,23) e Indice de Masa Corporal $-\mathrm{Z}$ score para edad y sexo (OR 2,04). El análisis multivariado muestra valores similares con $\mathrm{p} \leq 0,001$. El estado nutricional de la madre no resultó un factor predisponente significativo en ninguno de los modelos.

Tabla 4. Predictores de clasificación materna errónea del estado nutricional de sus hijos.

\begin{tabular}{|c|c|c|c|c|c|c|}
\hline \multirow{2}{*}{ Variables } & \multicolumn{3}{|c|}{ Análisis bivariado } & \multicolumn{3}{|c|}{ Análisis multivariado } \\
\hline & Odds Ratio & IC $95 \%$ & p-valor & Odds Ratio & IC $95 \%$ & p-valor \\
\hline \multicolumn{7}{|l|}{ Sexo } \\
\hline Femenino & 1 & - & 0.009 & 1 & & \\
\hline Masculino & 1.54 & $1.11-2.13$ & & 1.51 & $1.07-2.14$ & \\
\hline \multicolumn{7}{|l|}{ Edad (años) } \\
\hline $4 \mathrm{a}<9$ & 1.92 & $1.24-2.98$ & & 1.64 & $1.03-2.64$ & \\
\hline $9 a<14$ & 1.23 & $0.79-1.92$ & $<0.001$ & 1.11 & $0.69-1.78$ & $8<0.001$ \\
\hline$? 14$ & 1 & - & & 1 & & \\
\hline $\begin{array}{l}\text { Estado Nutricional } \\
\text { de pacientes (BAZ) }\end{array}$ & 2.04 & $1.74-2.40$ & $<0.001$ & 2.02 & $1.72-2.38$ & \\
\hline $\begin{array}{l}\text { Estado Nutricional } \\
\text { de la Madre (IMC) }\end{array}$ & 1.03 & $0.99-1.07$ & 0.078 & - & - & \\
\hline
\end{tabular}

\section{DISCUSIÓN}

En este estudio, casi $60 \%$ de las madres clasificaron el estado nutricional de sus hijos de manera correcta. Sin embargo, sólo el $21 \%$ de ellas clasificó correctamente a su hijo con sobrepeso y menos aún, sólo el 9,7\% clasificó correctamente a su hijo como obeso. Este porcentaje es similar a varios estudios publicados ${ }^{(10,18,19)}$, no así comparado con el estudio nacional representativo de Estados Unidos, donde, por el contrario, el 2/3 de las madres reconoció a su hijo con sobrepeso u obesidad como tal ${ }^{(16)}$.

El hallazgo de que a medida que aumenta el índice de masa corporal, es decir, en la medida que los hijos presenten sobrepeso u obesidad, las probabilidades de discordancia son mayores, puede reflejar la dificultad que tienen las madres para reconocer el sobrepeso de su propio hijo, una resistencia a admitir que su hijo tiene sobrepeso, o la falta de entendimiento de lo que significan tanto el sobrepeso como la obesidad. Según este estudio, algunos factores significativos que pudieren incidir en la clasificación errónea del estado nutricional de 
los niños por sus madres son el sexo masculino, menor edad y mayor índice de masa corporal. Un estudio realizado en Estados Unidos, de Jain y colaboradores $^{(11)}$ provee evidencia cualitativa que aquellas madres con menores ingresos económicos se niegan a describir a su hijo pre-escolar como con sobrepeso. Estas madres manifestaron su disconformidad con las tablas de crecimiento, restándole importancia. La tendencia de estas madres a minimizar los factores de riesgo asociados con el sobrepeso podría también ser extrapoladas a otros estratos sociales y niveles educativos, sin embargo, más estudios son necesarios al respecto.

Otro aspecto que llama la atención es la mayor discordancia a menor edad de los hijos. Volviendo al estudio de Jain y colaboradores ${ }^{(11)}$, las madres afirman confiar en que, en la medida que su hijo crezca, va a ir ganando altura y, por tanto, volver a un índice de masa corporal adecuado. Sin embargo, también manifiestan tener en cuenta que esto puede no suceder.

Los niños con sobrepeso y obesidad sólo fueron clasificados correctamente por sus madres sólo en un $31 \%$ de los casos. Son éstos los pacientes con mayor riesgo de presentar complicaciones secundarias $^{(20,21)}$ tales como presión arterial elevada, dislipidemias, resistencia a la insulina, diabetes, problemas ortopédicos, además de desórdenes psicológicos y emocionales. Desde el punto de vista clínico, resulta crítico reconocer este grupo de pacientes para poder ahondar en el despistaje de los factores de riesgo y tratarlos de ser necesario o derivarlos a atención especializada.

Así como las madres definen mejor el estado nutricional de sus hijos mayores de 14 años, también definen mejor el estado nutricional de sus hijas

\section{REFERENCIAS BIBLIOGRÁFICAS}

1. Ogden CL, Flegal KM, Carroll MD, Johnson CL. Prevalence and trends in overweight among US children and adolescents, 1999-2000. JAMA. 2002; 288:1728-1732. mujeres, en concordancia con varios estudios que demuestran el mayor interés de la madre en crear un ambiente de alimentación saludable y recalcar la importancia de la buena nutrición y ejercicio a sus niñas ${ }^{(22-24)}$. Por otro lado, son también las mujeres quienes sienten una mayor presión social a verse más delgadas, incluso a niveles más extremos de trastornos de la conducta alimentaria, más frecuente en mujeres.

El reconocimiento por los padres del sobrepeso y obesidad infantil es un paso fundamental tanto para la prevención como para el tratamiento del exceso de peso y sus consecuencias ${ }^{(25)}$. Sin embargo, los pediatras no estamos exentos de responsabilidad y debemos realizar el diagnóstico oportuno y guiar a los pacientes a un tratamiento multidisciplinario.

\section{CONCLUSIÓN}

Aproximadamente el $60 \%$ de las madres clasifican el estado nutricional de sus hijos de manera correcta. Sin embargo, los niños con sobrepeso y obesidad son reconocidos por sus madres como tales sólo en un $31 \%$ de los casos.

Se considera estos hallazgos como de gran importancia para el éxito de los programas de intervención en obesidad, ya que si las madres no reconocen el sobrepeso de sus hijos, éstos tienden a fracasar.

Los pediatras juegan un papel muy importante en determinar quiénes son las madres que no reconocen el sobrepeso de sus hijos, y por tanto, los factores de riesgo asociados, y constituyen un eslabón primordial al pesar y medir a los pacientes y concienciar a los padres sobre los beneficios de llevar una vida saludable en familia.

2. Dietz WH. Health consequences of obesity in youth: childhood predictors of adult disease. Pediatrics. 1998; 101:518-525. 
3. Must A, Strauss RS. Risks and consequences of childhood and adolescent obesity. Int J Obes Relat Metab Disord. 1999; 23:S1-S11.

4. Caprio S, Hyman LD, McCarthy S, Lange R, Bronson M, Tamborlane WV. Fat distribution and cardiovascular risk factors in obese adolescent girls: importance of the intraabdominal fat depot. Am J Clin Nutr. 1996; 64:12-17.

5. Freedman DS, Dietz WH, Srinivasan SR, Berenson GS. The relation of overweight to cardiovascular risk factors among children and adolescents: the Bogalusa Heart Study. Pediatrics. 1999; 103:1175-1182.

6. Pinhas-Hamiel O, Dolan LM, Daniels SR, Standiford D, Khoury PR, Zeitler P. Increased incidence of non-insulindependent diabetes mellitus among adolescents. J Pediatr. 1996; 128:608-615.

7. Kinugasa A, Tsunamoto K, Furukawa N, Sawada T, Kusunoki T, Shimada N. Fatty liver and its fibrous changes found in simple obesity of children. J Pediatr Gastroenterol Nutr. 1984;3:408-414.

8. Mallory GB Jr, Fiser DH, Jackson R. Sleep-associated breathing disorders in morbidly obese children and adolescents. J Pediatr. 1989; 115:892-897.

9. Dietz WH, Gross WL, Kirkpatrick JA. Blount disease (tibia vara): another skeletal disorder associated with childhood obesity. J Pediatr. 1982; 101:735-737.

10. Baughcum AE, Chamberlin LA, Deeks CM, Powers SW, Whitaker RC. Maternal perceptions of overweight preschool children. Pediatrics. 2000; 106:1380-1386.

11. Jain A, Sherman SN, Chamberlin DL, Carter Y, Powers SW, Whitaker RC. Why don't low-income mothers worry about their preschoolers being overweight?. Pediatrics. 2001; 107:1138-1146.

12. Flores-Peña $\mathrm{Y}$, Camal-Ríos N, Cerda-Flores R. Evaluación de la percepción materna del peso del hijo y de la heredabilidad del IMC en diadas mestizas del Sureste de México. Arch Latinoam Nutr. 2011; 61(4):389-95.

13. Cabrera-Rojas N, Rolón-Arambulo R, Garcete-Mañotti L, Sanabria M, Arredondo M, Pizzarro F. Concordancia entre la percepción materna y el estado nutricional real de niños preescolares que asisten a la consulta de pediatría general. Pediatría (Asunción). 2013;40(3):235-40.

14. Flores-Peña Y, Trejo-Ortiz P, Gallegos-Cabriales E, Cerda-Flores R. Validez de dos pruebas para evaluar la percepción materna del peso del hijo. Salud Pública México. 2009; 51(0):489-95.

15. Binkin N, Spinelli A, Baglio G, Lamberti A. Lo que es común se vuelve normal: el efecto de la prevalencia de la obesidad en la percepción materna. Nutr Metab Cardiovasc Dis. 2011;23(5):410-6.

16. Michele L, Galuska D, Blanck H, Serdula M. Maternal Perceptions of Weight Status of Children. Pediatrics. 2003; 111:1226-1231.

17. Sarmiento Quintero F, Ariza AJ, Barboza García F, Canal de Molano N, Castro Benavides M, Cruchet Muñoz S, et al. Sobrepeso y obesidad: revisión y puesta al día. Acta Gastroenterol Latinoam. 2016; 46:131-159.

18. Jackson J, Strauss CC, Lee AA, Hunter K. Parents' accuracy in estimating child weight status. Addict Behav. 1990; 15:65-68.

19. Young-Hyman D, Herman LJ, Scott DL, Schlundt DG. Care giver perception of children's obesity-related health risk: a study of African American families. Obes Res. 2000; 8:241-248

20. Barlow SE, Dietz WH. Obesity evaluation and treatment: Expert Committee recommendations. Pediatrics. 1998; 102(3).

21. Himes JH, Dietz WH. Guidelines for overweight in adolescent preventive services: recommendations from an expert committee. The Expert Committee on Clinical Guidelines for Overweight in Adolescent Preventive Services. Am J Clin Nutr.1994; 59:307-316.

22. Hill AJ, Pallin V. Dieting awareness and low self-worth: related issues in 8-year-old girls. Int J Eat Disord. 1998; 24:405-413.

23. Davison KK, Birch LL. Weight status, parent reaction, and self-concept in five-year-old girls. Pediatrics. 2001; 107:46-53.

24. Faith MS, Leone MA, Ayers TS, Heo M, Pietrobelli A. Weight criticism during physical activity, coping skills, and reported physical activity in children. Pediatrics. 2002; 110(2).

25. Watkins M, Clark K, Foster C, Welch K, Kasa-Vubu J. Relationships among Body Mass Index, Parental Perceptions, Birthweight and Parental Weight after referral to a Weight Clinic. Jour Nat Med Associat. 2007; 99:8. 